Donkervoort, M., Dekker, J., Stehmann-Saris, F.C., Deelman, B.G. Efficacy of strategy training jin left hemisphere stroke patients with apraxia: a randomised clinical trial. Neuropsychological

\begin{tabular}{|l|l|}
\hline $\begin{array}{l}\text { Postprint } \\
\text { Version }\end{array}$ & 1.0 \\
\hline Journal website & http://www.tandfonline.com/doi/abs/10.1080/09602010143000093 \\
\hline Pubmed link & \\
\hline DOI & $10.1080 / 09602010143000093$ \\
\hline
\end{tabular}

This is a NIVEL certified Post Print, more info at http://www.nivel.eu

\title{
Efficacy of strategy training in left hemisphere stroke patients with apraxia: A randomised clinical trial
}

\section{MiREILLE DONKERVOORT}

Netherlands Institute for Health Services Research, Utrecht, The Netherlands JOOST DEKKER

Netherlands Institute for Health Services Research and Vrije Universiteit, Amsterdam, The Netherlands

FIENEKE C. STEHMANN-SARIS

University of Professional Education, Amsterdam, The Netherlands BETTO G. DEELMAN

University of Groningen, The Netherlands

The objective of the present study was to determine in a controlled study the efficacy of strategy training in left hemisphere stroke patients with apraxia. A total of 113 left hemisphere stroke patients with apraxia were randomly assigned to two treatment groups; (1) strategy training integrated into usual occupational therapy and (2) usual occupational therapy only. Assessments took place at baseline, after an 8 week treatment period and 5 months after baseline (followup).

Patients were assessed on apraxia, motor functioning and activities of daily living (ADL). The primary outcome measure was a standardised ADL observation by a blinded research assistant. Additional ADL measures were used as secondary outcome measures (Barthel ADL index, ADL judgement by occupational therapist and by patient).After 8weeks of treatment, patients who received strategy training $(n=43)$ improved significantly more than patients in the usual treatment group $(\mathrm{n}=39)$ on the ADL observations (mean change .24; 90\% CI, .15-.34 vs. .12, .03-.21).This reflects a small tomediumeffect (effect size .37) of strategy training on ADL functioning. With respect to the secondary outcome measures a medium effect (effect size .47) was found on the Barthel ADL index. 
Donkervoort, M., Dekker, J., Stehmann-Saris, F.C., Deelman, B.G. Efficacy of strategy training in left hemisphere stroke patients with apraxia: a randomised clinical trial. Neuropsychological Rehabilitation: 2001, 11(5), 549-566

No beneficial effects of strategy training were found after 5 months (at followup).

In this trial evidence was found for the short-term effectiveness of strategy training in left hemisphere stroke patients with apraxia.

\section{INTRODUCTION}

Approximately half of all individuals who survive a stroke are disabled because of persisting neurological impairments (Brandstater, 1990). The patient often has hemiplegia, but the consequences may also include perceptual and cognitive impairments. One of the cognitive impairments following stroke that can have a major impact on independence in activities of daily living, is apraxia.

Apraxia is the inability to carry out learned and purposeful activities. This inability cannot be explained by primary motor or sensory impairments, or deficits in motivation, memory or comprehension (Kolb \& Wishaw, 1990; Rothi \& Heilman, 1997). A patient with apraxia does not know what to do, because the plan of action is disrupted, or in other cases, the patient knowswhat to do but not how to do it (De Renzi, 1989). It is estimated that approximately $30 \%$ of patients with a left hemisphere stroke have apraxic impairments (De Renzi, 1989; Donkervoort et al., 2000).

Patients with apraxia are frequently referred to occupational therapists. Van Heugten et al. developed a standardised occupational therapy programme for left hemisphere stroke patients with apraxia (Van Heugten et al., 1998, 1999a, 2000a). Themain principle of this therapy programme is the use of strategies to compensate for the apraxic impairment during the performance of daily living activities. Applying strategies to compensate for the presence of apraxia is expected to maximise the patient's independence. This approach was based on the positive results of strategy training programmes for memory and attention deficits (Berg, Koning-Haanstra \& Deelman, 1991; Fasotti, Kovacs, Eling, \& Brouwer, 2000).

Van Heugten et al. (1998) evaluated their therapy programme in a pre-post test design study with 33 left hemisphere stroke patients with apraxia. Their patients showed large improvements in ADL functioning and small improvements on apraxia and motor functioning. These results suggested that the programme was successful in teaching patients compensatory strategies, which enabled them to function more independently, despite the lasting presence of apraxia. Since this was an uncontrolled open study conclusions remained tentative.

The goal of the present study was to determine in a controlled study the efficacy of strategy training in left hemisphere stroke patientswith apraxia. Themain expectations to be tested were that (1) strategy training incorporated into usual occupational therapywill lead tomore independence of ADL functioning than usual treatment alone; while (2) there will be no differential effect with regard to the apraxic impairment itself. In addition, it is expected (3) that usual treatment without strategy training will lead to more improvement in motor functioning, because more time is available for training of motor functions. 
Donkervoort, M., Dekker, J., Stehmann-Saris, F.C., Deelman, B.G. Efficacy of strategy training ith left hemisphere stroke patients with apraxia: a randomised clinical trial. Neuropsychological Rehabilitation: 2001, 11(5), 549-566

\section{METHODS}

\section{Patients}

From November 1996 until July 1999 participating occupational therapists of 49 Dutch institutions (15 rehabilitation centres and 34 nursing homes) reported every consecutive patient with a left hemisphere stroke referred for occupational therapy. Criteria for the inclusion of patients into the present study were: (1) a left hemisphere stroke; (2) apraxia; and (3) staying on an inpatient care unit. Exclusion criteria were: (1) a history of apraxia before current stroke; (2) stroke had occurred less than 4weeks ormore than 2 years ago; (3) age younger than 25 years or older than 95 years; (4) a history of traumatic brain damage in combination with a period of coma lasting longer than 15 min or a period of post-traumatic amnesia existing longer than 2 hours; (5) a history of brain tumour; (6) no working knowledge of the Dutch language; (7) premorbid or present pathologies such as a psychiatric or psychogeriatric history, addiction to alcohol, medical or other drugs; (8) premorbid personality, intellectual or learning disorders; (9) a history of severe consciousness impairments; and (10) the treating physician, the occupational therapist, and/or the patient did not judge the treatment of apraxia to be necessary.

A left hemisphere stroke was diagnosed when acute clinical symptoms of a focal dysfunction of the left hemisphere were present; these signs and symptoms lasted at least 24 hours, and they were of a vascular origin only (WHO, 1989). The diagnosis of apraxia involved two steps. First, a clinical diagnosis of apraxia was made by the patient's treating medical team. The patient was diagnosed as apraxic if (1) the patient showed an inability (or restriction in the ability) to carry out purposeful activities and (2) this inability was not the result of a primary motor or sensory impairment, or deficit of comprehension or motivation (De Renzi, 1989; Kolb \& Wishaw, 1990).

Second, before final inclusion in the study, patients were tested by a trained researcher for severity of apraxia (Van Heugten et al., 1999b). Patients who showed no or minimal apraxic symptoms on the test (score above 87 ) were excluded from the study.

All patients (and their families) received verbal and written information about the study and gave verbal as well as written consent to participate.

\section{Study design}

A randomised, single blind, controlled trial design was used to compare the effect of strategy training (integrated into usual occupational therapy) with the effect of usual occupational therapy alone. Patientswere followed over a period of 5 months. During the first 8 weeks of this period patients received treatment according to their randomisation. Baseline measurements were done immediately after inclusion (except for the apraxia test which was part of the inclusion procedure). After the 8 week treatment period, a second measurement took place and the final measurement was a follow-up five months after the first assessment (Figure 1). Treatment of patients during the follow-up period was not prescribed in specific study guidelines. The study protocol was approved by the ethics committees of all participating institutions.

\section{Randomisation}


Donkervoort, M., Dekker, J., Stehmann-Saris, F.C., Deelman, B.G. Efficacy of strategy training jin left hemisphere stroke patients with apraxia: a randomised clinical trial. Neuropsychological

Occupational therapists.

In each institution the participating occupational therapists were randomly assigned to one of the two treatment regimens according to the principle of random permuted blocks; blocks of two were used (Pocock, 1983).

Patients.

After inclusion patients were randomly assigned to either strategy straining (integrated into usual occupational therapy) or usual treatment by a researcher using sequential numbered, not transparent, sealed envelopes. Patients were pre-stratified on type of institution (rehabilitation centre/ nursing home), time since stroke (more than 10 weeks/less than 10 weeks), and score on the apraxia test (more than 67/less than 67) in order to achieve comparability regarding these factors. A randomisation list was prepared for each stratum (using a random number table) according to the principle of random permuted blocks (of two patients) within strata (Altman, 1993; Pocock, 1983). The Zelen correction (Pocock, 1983; Zelen, 1974) was used to prevent an unequal distribution of patients between the two interventions within each institution and by that to control for differences between institutions such as amount of therapy and content of usual occupational therapy.

\section{Blinding}

The primary outcome assessments were carried out by a blinded research assistant. Because of the type of intervention, it was not possible to blind therapists or patients with regard to treatment. However, patients were not informed about the assigned intervention. Because they were not familiar with occupational therapy, they were naive with regard to their treatment as being strategy training or not. After the 8 week treatment period it was checked whether the research assistant or patient knew which treatment the patient had received.

\section{Interventions}

The occupational therapists in the strategy training group received additional training consisting of a full day course followed by several booster meetings.

Differences between institutions with regard to the amount of treatment, the content of the usual occupational therapy and treatment outside the occupational therapy departmentwere controlled for by the randomisation procedure.

\section{Strategy training}

Strategy training consisted of the treatment programme for left hemisphere stroke patients with apraxia, developed in a prior study (Van Heugten et al., 1998). Themain principle of this programme is the use of strategies to compensate for the apraxic impairment during the performance of activities in daily living. Patients are taught strategies to compensate internally or, if necessary, externally for the impairment. Examples are self-verbalisation to support the performance and writing down or showing pictures of the proper sequence of activities. The treatment aims at gradually teaching the patient more efficient strategies. A detailed diagnostic assessment of disability is the starting point of the treatment. The assumption underlying this programme is that, although recovery is possible in some cases, the apraxic impairment is frequently irreversible. Therefore treatment should focus on teaching patient ways to improve their ADL functioning, by learning strategies to compensate for the apraxic impairment. 
Donkervoort, M., Dekker, J., Stehmann-Saris, F.C., Deelman, B.G. Efficacy of strategy training inh left hemisphere stroke patients with apraxia: a randomised clinical trial. Neuropsychological Rehabilitation: 2001, 11(5), 549-566

\section{Usual treatment}

Usual occupational therapy concentrates on (sensory)motor, perceptual and cognitive deficits of the stroke patient and aims at increasing independent functioning inADL tasks (Trombly, 1989). In general, themain focus of the therapy is on (sensory) motor impairments (muscle tone, reflexes, controlled movements, muscle strength, contractures) and disability due to these impairments.

A variety of treatment methods is mentioned in the literature and used in daily practice (Bobath, 1977; Brunnstrom, 1970; Trombly, 1989).

\section{Measurements}

\section{ADL functioning}

The ADL observations (Van Heugten et al., 1999a, 2000a) are a set of standardised observations specially developed to assess disabilities due to apraxia.

The performance on four standardised tasks is observed (1) wash the face and upper body, (2) put on a shirt or blouse, (3) prepare and eat a sandwich, and (4) prepare a cup of hot chocolate. Scores are assigned for both the level of independence and the nature and severity of the restrictions in the performance.

The overall mean score (used in this study) ranges from totally dependent (score 0) to totally independent (score 3). The internal consistency and interobserver reliability of this observation procedure were found to be good (Van Heugten et al., 1999a, 2000a).

Besides the standardised specific ADL observations three other general

ADLmeasureswere used: the Barthel ADL Index (Wade\&Collin, 1988) and a more extendedADL judgement list filled in by the occupational therapist (OT) and an identical list filled in by the patient (Pt). The ADL judgement list is based on the Rivermead Activities of Daily Living Scale (Lincoln \& Edmans, 1990) and consists of 38 various daily activity items (ranging from eating to making use of public transport). All items are scored on a scale from totally independent (score 0 ) to totally dependent (score 10). The overallmean score is used in this study. The internal consistency of both the ADL judgement (OT) and the ADL judgement (Pt) is good: Cronbach's alpha in the present study is .93 and .92, respectively.

\section{Apraxia}

The Apraxia Test is based on a test of De Renzi (DeRenzi, 1989; Van Heugten et al., 1999b) and consists of two subtests assessing the ability to use objects or pantomime use of objects and the ability to imitate gestures (with the not affected ipsilateral arm). Themaximumscore of the total test is 90 , whichmeans that the patient does not show any apraxic symptoms on the test. The internal consistency and the validity of the test is good (Van Heugten et al., 1999b).

\section{Motor functioning}

The Motricity Index (Demeurisse, Demol, \& Robaye, 1980) measures voluntary movements of the limbs on the affected contralateral side. The test consists of six items (three arm/hand and three leg/feet) and the maximum side score is 100 . The Motricity Index has been shown to have good validity and reliabilitywith stroke patients (Collin\&Wade, 1990; Demeurisse et al., 1980). 
Donkervoort, M., Dekker, J., Stehmann-Saris, F.C., Deelman, B.G. Efficacy of strategy training inh left hemisphere stroke patients with apraxia: a randomised clinical trial. Neuropsychological Rehabilitation: 2001, 11(5), 549-566

The Functional Motor

Test is a simplified version of the Action Research Arm Test (Lyle, 1981) and measures the voluntary functional activity of the armand hand of the affected contralateral side. It consists of four items in which the patient has to use a type of grip (pinch, grip, or grasp) to manipulate an object (pick it up,move it forward and put it down again). Themaximum score for the total test is 12. The internal consistency is good, Cronbach's alpha in the present study is .88 .

\section{Additional measures}

Standardised tests assessing verbal comprehension (SAN: Deelman, Liebrand, Koning-Haanstra \& van de Burg, 1987),memory (Five Picture Test: Van der Molen \& Deelman, 1986), neglect (Star Cancellation Test: Wilson, Cockburn, \& Halligan, 1987) and mental status (Cognitive Screening Test: De Graaf \& Deelman, 1991) were used to monitor the patients' general cognitive functioning during the study. Occupational therapists completed a questionnaire (at baseline) on demographic and clinical, stroke-related data and a questionnaire (at post-treatment and follow-up assessment) concerning the treatment patients received during the study, such as the amount and content of occupational therapy and other therapies received (physical therapy, speech therapy, etc).

\section{Statistical analysis}

The aimof the studywas to examine the effect of strategy training on independence in activities of daily living. Therefore, the objective ADL observations were chosen as the primary outcome measure. The other measures of ADL functioning were used as secondary outcome measures (Barthel and ADL judgement). A sample size of 50 patients for each intervention group was required to detect amedium and clinically relevant effect size of .5 on the ADL observations with a significance level of .05 and a power of $80 \%$ (Cohen, 1988). Analyses were based on the patients as randomised, known as the intention-to-treat principle. In addition, a per-protocol analysis was performed excluding patients with deviations from the treatment protocol.

Between group differences at baseline were investigated by chi-square or $t$-test where appropriate. In order to evaluate outcome, change scores were calculated by subtracting baseline scores from post-treatment and follow-up scores, respectively. The change scores were compared between the two interventions.

Analysis of covariancewas used to test for between group differences in change scores. Adjusted analyses were performed: The baseline score of each outcome measure was included as a covariate in order to improve precision in controlling for influence of baseline score on outcome. The two intervention groups differed at baseline with respect to age (see Results section), therefore age was also included as a covariate in the analyses. A one-sided significance test (significance level .05) was used corresponding the expectation that strategy training would have a positive effect on ADL functioning.

Mean differences between groups and $90 \%$ confidence intervals were calculated for all outcome measures. In addition, effect sizes were calculated, dividing the mean difference between the interventions by the standard deviation of the change score of the total population. An effect size of .2 is regarded as small, of .5 as medium, and .8 as large (Cohen, 1988). 
Donkervoort, M., Dekker, J., Stehmann-Saris, F.C., Deelman, B.G. Efficacy of strategy training inh left hemisphere stroke patients with apraxia: a randomised clinical trial. Neuropsychological Rehabilitation: 2001, 11(5), 549-566

Finally, subgroup analyses were performed to study whether differences existed in the effects of strategy training between subgroups (rehabilitation centres/nursing homes; time since stroke more/less than 10 weeks; apraxia test score above/below median, score on ADL observations above/below median).

The interaction between type of treatment and subgroup was tested with analysis of covariance. Analyseswere carried out using SPSS 8.0 for Windows.

\section{RESULTS}

Figure 1 shows the patient flow and follow-up to week 20. During the study, 315 patients with apraxia were referred for occupational therapy in the participating institutions. Of these 315 patients, 176 patients were not eligible (Figure 1) and an additional 26 patients appeared to be ineligible during the final criteria check by the researcher. In total 113 patients with a left hemisphere stroke diagnosed as apraxic participated in the study.

\section{Comparability}

Occupational therapists in the two intervention groups did not differ significantly in age, gender, years of experience, amount of work hours per week and experience with patients with cognitive impairments. With respect to baseline characteristics, the patients are in general equally divided over the two intervention groups, except for age (Tables 1 and 2). Patients in the strategy training groupwere older. The number of dropouts, reasons for dropping out and demographic characteristics of dropouts were equally divided over the intervention groups. There was no relation between missing values on outcome measures (post-treatment) and test scores at baseline, except for apraxia. Patients with missing values at the post-treatment assessment more frequently had a poor score on the apraxia test at baseline. However, the baseline apraxia score of patients with missing values at the post-treatment assessment did not differ between the two treatment groups. Therefore, the comparison between the two interventions is not considered to be biased.

\section{Treatment}

The amount (sessions and hours) of occupational therapy that patients received did not differ significantly between the two intervention groups $(\mathrm{t}=-1.06, \mathrm{t}=-1.63, \mathrm{p}>$ .10). Patients in the strategy training group had on average 25 (SD 9.8) sessions, resulting in 15 (SD 7.7) hours of occupational therapy. The usual treatment group had on average 27 (SD 15.6) sessions and 19 (SD 15.0) hours of occupational therapy. Furthermore, the two intervention groups did not differ with respect to the number of patients receiving ADL training, training of impaired motor functions, cognitive training, education, splinting, advice and applications for aids, and housing adjustments. Although the number of patients receiving ADL training was equal in both groups, the percentage of treatment spent on ADL training was - as expectedsignificantly higher in the strategy training group $(t=2.12, p<.05)$. With respect to the use of other therapies we found no significant differences between the two groups. 
Donkervoort, M., Dekker, J., Stehmann-Saris, F.C., Deelman, B.G. Efficacy of strategy training inh left hemisphere stroke patients with apraxia: a randomised clinical trial. Neuropsychological Rehabilitation: 2001, 11(5), 549-566

\section{[FIGURE 1] [TABLE 1]}

It was not possible to give treatment according to the study protocol for seven patients; that is patients were assigned to one treatment group but did not receive that specific treatment, or they did not receive occupational therapy at all (Figure 1).

\section{Success of blinding}

The success of the blinding of the patients and the research assistant who performed the outcome measurements was checked after the 8 week treatment period. Analyses showed that they had not guessed the allocation correctly more often than wrongly (kappa $=.03$ and -.17 , respectively). The research assistant reported only nine disclosures of treatment allocation, unintentionally caused by remarks from occupational therapists (six strategy training vs. three usual treatment).

\section{[TABLE 2]}

\section{Outcome}

Table 3 shows that patients in the strategy training group improvedmore on the ADL observations (primary outcome measure) than patients in the usual treatment group, $F(1,81)=3.52, p=.03$. The mean ADL observation score in the strategy training group improved 0.24 over a period of 8 weeks and in the usual treatment group the ADL observation score improved 0.12. The corresponding effect size (.37) indicates that strategy training is associated with a small to medium effect on ADL functioning. Additionally, after controlling for improvement in apraxia and motor functioning the larger improvement on the ADL observations in the strategy training group is still significant, $F(1,80)=3.37, p=.04$.

\section{[TABLE 3]}

The change in ADL judgements by the occupational therapists and the patient (secondary outcome measures) did not show a difference in change frombaseline between the two intervention groups. The Barthel ADL index, on the other hand, did show a significant, medium effect (effect size .47) in favour of strategy training, $\mathrm{F}(1$, $92)=7.31, p<.01$. The intervention groups did not differ with respect to improvement in apraxia or motor functioning.

A per-protocol analysis was conducted, excluding all patients who were not treated according to the study protocol $(n=7)$. The results of the per-protocol analysis were similar to the results of the intention to treat analysis, for both primary, $F(1,77)=$ $3.64, p=.03$, and secondary outcome measures.

\section{Outcome at follow-up}

Table 4 shows the outcome measurements at follow-up for both intervention groups. No significant differences between the two intervention groups were found on any of the ADLmeasures. Also with respect to motor functioning and apraxia the two intervention groups do not differ at follow-up. Besides these measures, information was collected with regard to residence and use of therapy at time of follow-up. The two intervention groups did not differ in the amount of patients discharged, deceased 
Donkervoort, M., Dekker, J., Stehmann-Saris, F.C., Deelman, B.G. Efficacy of strategy training in left hemisphere stroke patients with apraxia: a randomised clinical trial. Neuropsychological Rehabilitation: 2001, 11(5), 549-566

or admitted to other institutions. Most patients still received therapy and although the two groups did not differ in use of therapy with respect to most therapies they did differ in the use of occupational therapy. More patients in the usual treatment group than in the strategy training group still received occupational therapy at follow-up ( $\left.\mathrm{c}_{2}=6.84, p=.01\right)$.

\section{Subgroup analysis}

Subgroup analyses with respect to the primary outcome measure were conducted to see if there are any specific effects of treatment in specific groups of patients. The results show subgroup differences with respect to the improvement on ADL observations suggesting strategy training to be more beneficial in patients treated in rehabilitation centres, in patients with a poor apraxia score and in patients with a poor score on ADL observations. However, none of the interactions between treatment and the subgroups was found to be significant $(0 \leq F \leq 1.2, p>.30)$.

\section{DISCUSSION}

In this trial evidence was found for effectiveness of strategy training in left hemisphere stroke patients with apraxia. The results show that after an 8 week treatment period strategy training integrated into usual occupational therapy is more effective in improving ADL functioning than usual occupational therapy alone. After a follow-up period the results show no differences between the two treatment groups. At follow-up, significantly more patients in the usual treatment group than in the strategy training group still received occupational therapy.

\section{[TABLE 4]}

The results confirm previous findings (Goldenberg \& Hagman 1998; Van Heugten et al., 1998) in which stroke patients with apraxia improved in ADL functioning after receiving occupational therapy. In the study by Van Heugten and colleagues, 33 left hemisphere stroke patients with apraxia were given strategy training and improved considerably with respect to ADL functioning while showing only small improvements in apraxia and motor functioning (Van Heugten et al., 1998). Goldenberg and Hagman (1998) trained ADL activities with 15 apraxia patients. In their training they combined assistance during the execution of an activity (in order to reduce the amount of errors) with training of details. Their therapy led to a significant improvement of trained ADL activities, while virtually no recovery was found when the same activities were not trained. These two studies did not use some of the methods regarded to be essential in studying the effect of therapy (e.g., individual patient randomisation, control subjects, blind assessment, pre-planned power, and adequate sample size). Thus, the results of the current trial confirm the results found in the earlier studies that used a less strong study design.

The clinical relevance of this study is the increase in independence of ADL functioning. In the literature quantitative measures of clinical relevance are suggested. Bronfort (1997) considered an effect size of .5 to be the threshold for minimal clinical importance. The beneficial effects we found on objectively observed ADL functioning and the Barthel ADL Index do not meet this stringent criterion. 
Donkervoort, M., Dekker, J., Stehmann-Saris, F.C., Deelman, B.G. Efficacy of strategy training ith left hemisphere stroke patients with apraxia: a randomised clinical trial. Neuropsychological Rehabilitation: 2001, 11(5), 549-566

However, it should be noted that in this study two therapies were compared (rather than a comparison between therapy and no therapy) and the intervention includes only a small part of the total rehabilitation process. Therefore, relatively small effects are to be expected. Thus, the small to medium effect sizes we found can certainly be considered to be of practical relevance.

It is of interest to note that the largest effect size was found in the Barthel ADL Index (a general measure of ADL functioning), rather than in the standardised ADL observations specifically designed to measure disabilities due to apraxia. This supports other papers that find that the Barthel ADL Index is perhaps the most sensitive measure of outcome available in rehabilitation research (Hocking, Williams, Broad, \& Baskett, 1999;Wade \& Collin, 1988; Wolfe, Taub, Woodrow, \& Burney, 1991).

We also studied whether there was a larger improvement in motor functioning in favour of the usual treatment group. After all, when not using strategy training more time would be available to train motor functions. The data show that the usual treatment group did not receive more training of motor functions and consistent with that, there was no difference with respect to improvement in motor functioning between the two treatment groups.

In the present study, appropriate randomisation was performed, selection criteria and patients' characteristics were clearly described, therapists were well-trained in using the written guidelines for strategy training, and the evaluation was performed by a blinded research assistant. Nevertheless, some methodological comments can be made. First, difficulties were encountered during the recruitment process. Two-thirds of the registered apraxia patients did not participate in the study, which makes generalisation difficult. However, many of the patients who did not participate would probably not have been trained intensively with respect to apraxia anyway, while two of the main reasons for not participating were (1) apraxia not severe enough to treat, or (2) the patient was in a poor condition. Second, no complete data were available on patients who withdrew from the study. Additional analyses on the number of withdrawals, reasons for withdrawal, and baseline scores of patients who withdrew did not indicate a selective bias with respect to the two intervention groups.

Third, not all patients were treated according to the assigned treatment $(n=7)$, resulting in a smaller treatment contrast than initially intended. An additional perprotocol analysis did not result in different conclusions than the intentionto- treat analysis. Finally, Van Heugten et al. (2000b) showed that the ADL observationsour primary outcome measure-display a ceiling effect.

Patients who function rather independently before the treatment period cannot improve during treatment. This ceiling effect could conceal the results of strategy training. Therefore, analyses were rerun without the patients who scored 2.75 or higher (approximately 20\%) on ADL observations at baseline.

This analysis confirmed the efficacy of strategy training, $F(1,63)=4.4, p=.02$. In addition to the post-treatment effects we also studied the long-term effectiveness of strategy training. The results show that the two intervention groups are comparable with respect to improvement in ADL functioning 5 months after initial participation in the study. So, no long-term effect in favour of strategy training was found. This was not due to a decline in ADL functioning in the strategy training group but due to a further improvement in ADL functioning in the usual treatment group. Furthermore, we found that significantly more patients in the usual treatment 
Donkervoort, M., Dekker, J., Stehmann-Saris, F.C., Deelman, B.G. Efficacy of strategy training inh left hemisphere stroke patients with apraxia: a randomised clinical trial. Neuropsychological Rehabilitation: 2001, 11(5), 549-566

group were still receiving occupational therapy at follow-up. These results suggest that patients in the usual treatment group needed more occupational therapy to obtain a corresponding level of improvement in ADL functioning compared to patients who received strategy training. With our data it is not possible to test this specific hypothesis. More research into the long-term effectiveness of strategy training is needed.

In conclusion, this randomised controlled trial showed beneficial effects of strategy training on ADL functioning in left hemisphere stroke patients with apraxia. The results suggest that the therapy programme is successful in teaching patients compensatory strategies, which enable themto function more independently, despite the lasting presence of apraxia.

\section{REFERENCES}

Altman, D.G. (1993). Practical statistics for medical research. London: Chapman \& Hall. Berg, I.J.,Koning-Haanstra , M.,\&Deelman, B.G. (1991). Long-termeffects of memory rehabilitation: A controlled study. Neuropsychological Rehabilitation, 1, 97-111.

Bobath, B. (1977). Treatment of adult hemiplegia . Physiotherapy, 63, 310-313.

Brandstater, M.E. (1990). An overview of stroke rehabilitation . Stroke, 21(suppl II), 40-42.

Bronfort, G. (1997). Efficacy of manual therapie s of the spine. (PhD. dissertation Vrije Universitei t Amsterdam, Institute for Research in Extramural Medicine.) Amsterdam: Thesis Publishers Amsterdam.

Brunnstrom, S. (1970). Movement therapy in hemiplegia. A neurophysiologica I approach. Hagerstown: Harper \& Row.

Cohen, J. (1988). Statistical power analysis for the behaviora I sciences. Hillsdale, NJ: Lawrence Erlbaum Associates.

Collin,C.,\&Wade,D. (1990). Assessing motor impairments after stroke:Apilot reliability study. Journal of Neurology, Neurosurgery and Psychiatry, 53, 576-579.

Deelman, B.G., Liebrand, W.B.G., Koning-Haanstra , M., \& Burg, W. van de. (1987). De SAN test: een afasietes $t$ voor auditief en mondeling taalgebruik : Handleiding. Lisse: Swets \& Zeitlinger.

De Graaf,A.,\&Deelman, B.G. (1991). De cognitieve screening test: handleiding .Lisse: Swets\& Zeitlinger.

Demeurisse, G., Demol, O., \& Robaye, E. (1980). Motor evaluation in vascular hemiplegia. European Neurology, 19, 382-389.

De Renzi, E. (1989). Apraxia. In F. Boller \& J. Grafman (Eds.), Handbook of neuropsycholog y (Vol. 2). Amsterdam: Elsevier.

Donkervoort, M., Dekker, J., Ende, E. van den, Stehmann-Saris, J.C., \& Deelman, B.G. (2000).

Prevalence of apraxia among patients with a first left hemisphere stroke in rehabilitatio $\mathrm{n}$ centres and nursing homes. Clinical Rehabilitation, 14, 130-136.

Fasotti, L., Kovacs, F., Eling, P.A.T.M.,\&Brouwer,W.H. (2000). Time pressure management as compensatory strategy training after closed head injury. Neuropsychologica I Rehabilitation, 10, 47-65.

Goldenberg, G., \& Hagman, S. (1998). Therapy of activities of daily living in patients with apraxia. Neuropsychological Rehabilitation, 8, 123-141.

Halligan, P., Cockburn, J., \& Wilson, B. (1991). The Behavioural assessment of visual neglect. Neuropsychological Rehabilitation, 1, 5-32.

Hocking, C., Williams, M., Broad, J. \& Baskett, J. (1999). Sensitivity of Shah, Vanclay and Cooper's modified Barthel Index. Clinical Rehabilitation , 13, 141-147.

Kolb, B.,\&Wishaw, I.Q. (1990). Fundamentals of human neuropsycholog y (3rd ed.).New York: Freeman.

Lincoln, N.B., \& Edmans, J.A. (1990). A re-validation of the Rivermead ADL Scale for elderly patients with stroke. Age \& Ageing, 19, 9-24. 
Donkervoort, M., Dekker, J., Stehmann-Saris, F.C., Deelman, B.G. Efficacy of strategy training in left hemisphere stroke patients with apraxia: a randomised clinical trial. Neuropsychological Rehabilitation: 2001, 11(5), 549-566

Lyle, R.C. (1981). A performance test for assessment of upper limb function in physical rehabilitatio $\mathrm{n}$ treatment and research. Internationa I Journal of Rehabilitation Research, 4 483-492.

Molen, L. van der,\&Deelman, B.G. (1986).De vijf afbeeldinge $n$ test als diagnostisc $h$ instrument bij het bepalen van dementie (scientific report). University of Groningen; Department of Neuropsychology.

Pocock, S.J. (1983). Clinical trials. Chichester: Wiley.

Rothi, L.J.G., \& Heilman, K.M. (1997). Apraxia: The neuropsycholog y of action. Hove, UK: Psychology Press.

Trombly, C.A. (1989). Occupational therapy for physical dysfunction. Baltimore: Williams \& Wilkins.

Van Heugten, C.M., Dekker, J., Deelman, B.G., Dijk, A..J. van, Stehmann-Saris , J.C., \& Kinebanian, A. (1998). Outcome of strategy training in stroke patients with apraxia: A phase- II study. Clinical Rehabilitation, 12, 294-303.

Van Heugten, C.M.,Dekker, J.,Deelman, B.G., Stehmann-Saris , J.C.,\&Kinebanian,A. (1999a).

Assessment of disabilities in stroke patients with apraxia: Internal consistency and interobserver reliability. Occupational Therapy Journal of Research, 19, 55-73.

VanHeugten, C.M., Dekker, J.,Deelman, B.G., Stehmann-Saris , J.C.,\&Kinebanian,A. (1999b).

A diagnostic test for apraxia in stroke patients: internal consistency and diagnostic value. The Clinical Neuropsychologis t, 13, 182-192.

Van Heugten, C.M., Dekker, J., Deelman, B.G., Dijk, A.J. van, Stehmann-Saris , J.C., \& Kinebanian, A. (2000a). Measuring disabilitie $s$ in stroke patients with apraxia: A validation study of an observational method. Neuropsychological Rehabilitation, 10, 401-414.

Van Heugten, C.M., Dekker, J., Deelman, B.G., Dijk, A..J. van, Stehmann-Saris J.C., \& Kinebanian, A. (2000b). Rehabilitation of stroke patients with apraxia: The role of additional cognitive and motor impairments. Disability \& Rehabilitation, 22, 547-554.

Wade, D.T., \& Collin, C. (1988). The Barthel ADL Index: A standard measure of physical disability? International Disability Studies, 10, 64-67.

Wilson, B.A., Cockburn, J., \& Halligan, P. (1987). Behavioural Inattention Test. Tichfield, Fareham, Hants, England: Thames Valley Test Co.; Gaylord, MI: National Rehabilitation Services.

Wolfe, C.D.A., Taub, N.A., Woodrow, E.J., \& Burney, P.G.J. (1991). Assessment of scales of disability and handicap for stroke patients. Stroke, 22, 1242-1244.

World HealthOrganization (1989). Special report: Recommendations on stroke prevention , diagnosis and therapy. Stroke, 20, 1407-1431.

Zelen, M. (1974). The randomizatio $n$ and stratificatio $n$ of patients to clinical trials. Journal of Chronic Diseases, 27, 365-375. 
Donkervoort, M., Dekker, J., Stehmann-Saris, F.C., Deelman, B.G. Efficacy of strategy training iin left hemisphere stroke patients with apraxia: a randomised clinical trial. Neuropsychologica

FIGURES AND TABLES

Figure 1. Flow chart of patients in the stroke-apraxia trial

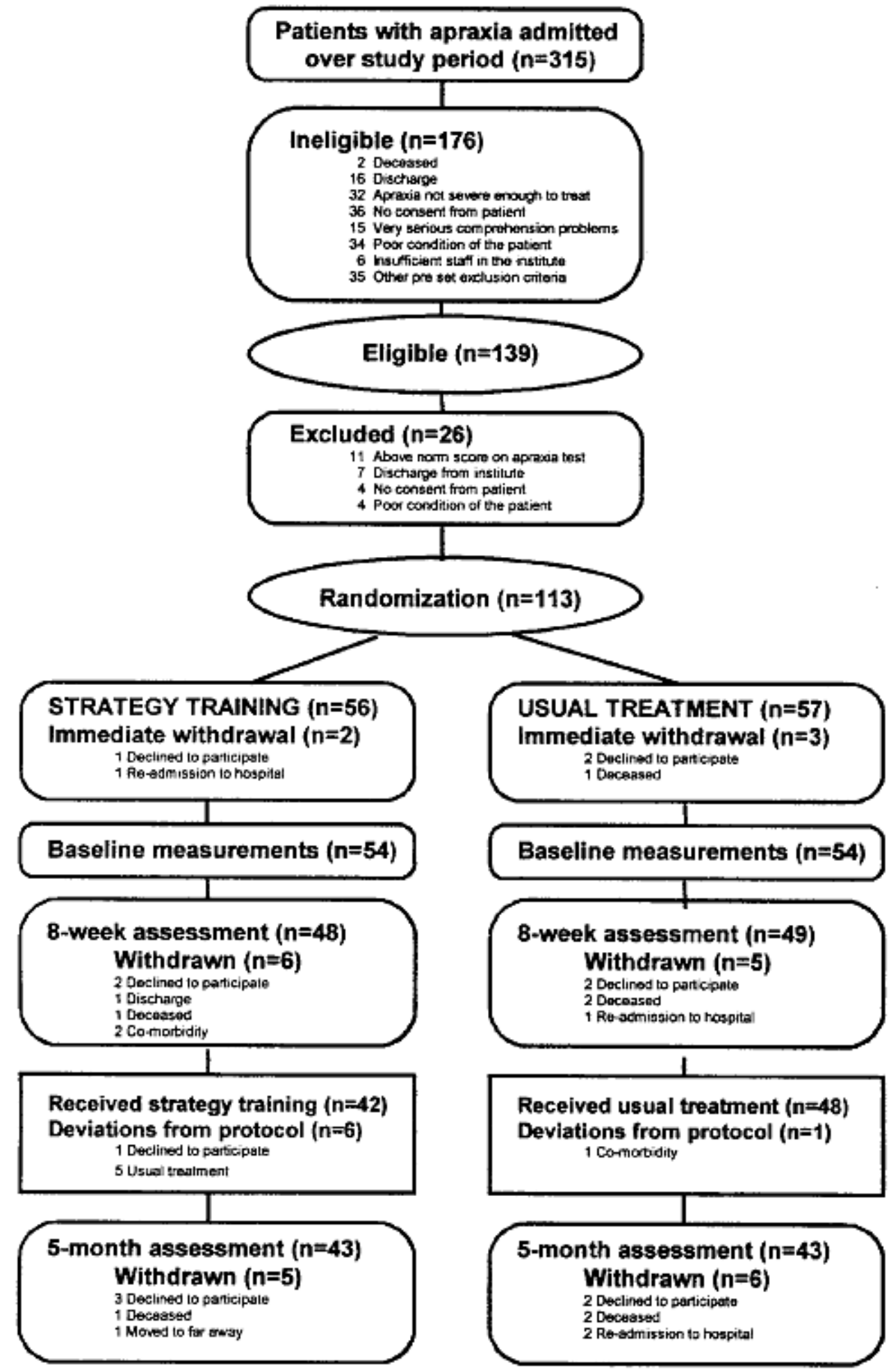


Donkervoort, M., Dekker, J., Stehmann-Saris, F.C., Deelman, B.G. Efficacy of strategy training ith left hemisphere stroke patients with apraxia: a randomised clinical trial. Neuropsychological

Rehabilitation: 2001, 11(5), 549-566

TABLE 1

Comparability of the intervention groups with respect to demographic and clinical characteristics $(n=113)$

\begin{tabular}{|c|c|c|}
\hline & $\begin{array}{l}\text { Strategy training } \\
\qquad(\mathrm{n}=56)\end{array}$ & $\begin{array}{l}\text { Usual treatment } \\
\qquad(\mathrm{n}=57)\end{array}$ \\
\hline \multicolumn{3}{|l|}{ Gender, $n(\%)$} \\
\hline Male & $29 / 56(52)$ & $35 / 57(61)$ \\
\hline Female & $27 / 56(48)$ & $22 / 57(39)$ \\
\hline Age at stroke*, mean (SD) & $67.6(11.7)$ & $63.3(11.6)$ \\
\hline \multicolumn{3}{|l|}{ Education, $n(\mathscr{\%})$} \\
\hline Low & $19 / 56(34)$ & $25 / 57(44)$ \\
\hline Middle & $17 / 56(30)$ & $12 / 57(21)$ \\
\hline High & $8 / 56(14)$ & $5 / 57 \quad(9)$ \\
\hline Unknown & $12 / 56(21)$ & $15 / 57(26)$ \\
\hline \multicolumn{3}{|l|}{ Residence before stroke, $n(\%)$} \\
\hline Living alone & $21 / 56(38)$ & $15 / 57(26)$ \\
\hline Living with partner and/or children & $34 / 56(61)$ & $38 / 57(67)$ \\
\hline Unknown & $1 / 56 \quad(2)$ & $4 / 57 \quad(7)$ \\
\hline Right handedness, $n(\%)$ & $5056(89)$ & $54 / 57(95)$ \\
\hline \multicolumn{3}{|l|}{ Type of institution, $n(\%)$} \\
\hline Rehabilitation centre & $39 / 56(70)$ & $37 / 57(65)$ \\
\hline Nursing home & $17 / 56(30)$ & $20 / 57(35)$ \\
\hline \multicolumn{3}{|l|}{ Type of stroke, $n(\%)$} \\
\hline Haemorrhage & $12 / 56(21)$ & $4 / 57 \quad(7)$ \\
\hline Infarction & $41 / 56(73)$ & $48 / 57(84)$ \\
\hline Otherwise/unknown & $3 / 56$ & $5 / 57 \quad(9)$ \\
\hline Time since stroke in days, mean (SD) & $100.2(63.3)$ & $102.9(70.7)$ \\
\hline Recurrent stroke, $n(\%)$ & $12 / 56(21)$ & $9 / 57(16)$ \\
\hline Hemiplegia/hemiparesis, $n(\mathscr{\%})$ & $5056(89)$ & $52 / 57(91)$ \\
\hline
\end{tabular}

* Difference between intervention groups, 2-tailed significance $p \leq .05$ 
Donkervoort, M., Dekker, J., Stehmann-Saris, F.C., Deelman, B.G. Efficacy of strategy training ith left hemisphere stroke patients with apraxia: a randomised clinical trial. Neuropsychological Rehabilitation: 2001, 11(5), 549-566

TABLE 2

Comparability of the intervention groups with respect to test results of motor, cognitive, and ADL functioning assessments at baseline

\begin{tabular}{|c|c|c|c|c|}
\hline & \multicolumn{2}{|c|}{$\begin{array}{l}\text { Strategy training } \\
\qquad(\mathrm{n}=54)\end{array}$} & \multicolumn{2}{|c|}{$\begin{array}{l}\text { Usual treatment } \\
\qquad(\mathrm{n}=54)\end{array}$} \\
\hline & $\mathrm{n}$ & $\operatorname{Mean}(S D)$ & $\mathrm{n}$ & $\operatorname{Mean}(S D)$ \\
\hline \multicolumn{5}{|l|}{ Motor functioning } \\
\hline Motricity Index & 53 & $50.8(31.3)$ & 54 & $40.9(30.1)$ \\
\hline Functional Motricity Test & 53 & $6.5 \quad(3.9)$ & 54 & $5.3(3.5)$ \\
\hline \multicolumn{5}{|l|}{ Cognitive functioning } \\
\hline Apraxia & 54 & $57.3(21.2)$ & 54 & $62.0(17.9)$ \\
\hline Verbal Comprehension & 47 & $32.9 \quad(8.7)$ & 53 & $32.6(6.6)$ \\
\hline Cognitive Screening Test & 47 & $13.2(4.7)$ & 49 & $13.0(4.0)$ \\
\hline 5-AT (memory) & 49 & $9.1(1.4)$ & 51 & 9.4 (1.1) \\
\hline Star cancellation (neglect) & 46 & $7.7(11.4)$ & 51 & $5.6(8.6)$ \\
\hline \multicolumn{5}{|l|}{ ADL functioning } \\
\hline Barthel & 53 & $10.7(4.9)$ & 53 & $11.2(5.0)$ \\
\hline ADL observations & 51 & $2.2(0.5)$ & 50 & $2.3(0.4)$ \\
\hline ADL judgement (OT) & 53 & 3.2 (1.3) & 53 & 3.1 (1.4) \\
\hline ADL judgement (Pt) & 43 & $4.1 \quad(1.5)$ & 51 & $4.4 \quad(1.6)$ \\
\hline
\end{tabular}

TABLE 3

Post-treatment outcome measures

\begin{tabular}{|c|c|c|c|c|c|c|c|}
\hline & \multicolumn{4}{|c|}{ Change from baseline } & & & \\
\hline & \multicolumn{2}{|c|}{$\begin{array}{l}\text { Strategy training } \\
\qquad(\mathrm{n}=48)\end{array}$} & \multicolumn{2}{|c|}{$\begin{array}{l}\text { Usual treatment } \\
\qquad(\mathrm{n}=49)\end{array}$} & \multicolumn{3}{|c|}{$\begin{array}{c}\text { Difference } \\
\text { between groups }\end{array}$} \\
\hline & $\mathrm{n}$ & Mean $(90 \% \mathrm{Cl})$ & $\mathrm{n}$ & $\operatorname{Mean}(90 \% \mathrm{Cl})$ & $(90 \% \mathrm{CI})$ & P value & Effect size \\
\hline \multicolumn{8}{|l|}{ Motor functioning } \\
\hline Motricity Index & 44 & $2.61(-0.26,5.49)$ & 49 & $2.98(0.09,5.87)$ & $-0.37(-4.41,3.68)$ & 0.39 & -0.03 \\
\hline Functional Motricity Test & 45 & $0.60(0.16,1.04)$ & 49 & $0.33(0.06,0.59)$ & $0.27(-0.23,0.77)$ & 0.13 & 0.18 \\
\hline \multicolumn{8}{|l|}{ Cognitive functioning } \\
\hline Apraxia & 44 & $4.20(1.69,6.72)$ & 48 & $2.21(-0.32,4.74)$ & $2.00(-1.54,5.53)$ & 0.25 & 0.20 \\
\hline \multicolumn{8}{|l|}{ ADL functioning } \\
\hline ADL observations & 43 & $0.24(0.15,0.34)$ & 39 & $0.12(0.03,0.21)$ & $0.13 \quad(0.00,0.25)$ & 0.03 & 0.37 \\
\hline Barthel ADL Index & 45 & $2.44(1.70,3.19)$ & 48 & $1.15(0.55,1.74)$ & $1.30(0.36,2.24)$ & 0.00 & 0.47 \\
\hline ADL judgement (OT) & 47 & $0.90(0.65,1.25)$ & 48 & $1.04(0.78,1.31)$ & $-0.14(-0.51,0.22)$ & 0.48 & -0.13 \\
\hline ADL judgement $(\mathrm{Pt})$ & 38 & $0.65(0.35,0.95)$ & 44 & $0.54(0.27,0.82)$ & $0.11(-0.29,0.51)$ & 0.25 & 0.10 \\
\hline
\end{tabular}

* Analyses are adjusted for age and baseline scores on the specific outcome measures; a one-sided significance test is used. 
Donkervoort, M., Dekker, J., Stehmann-Saris, F.C., Deelman, B.G. Efficacy of strategy training ith left hemisphere stroke patients with apraxia: a randomised clinical trial. Neuropsychological

Rehabilitation: 2001, 11(5), 549-566

TABLE 4

Follow-up outcome measures

\begin{tabular}{|c|c|c|c|c|c|c|c|}
\hline & \multicolumn{4}{|c|}{ Change from baseline } & & & \\
\hline & \multicolumn{2}{|c|}{$\begin{array}{l}\text { Strategy training } \\
\quad(\mathrm{n}=43)\end{array}$} & \multicolumn{2}{|c|}{$\begin{array}{l}\text { Usual treatment } \\
\qquad(\mathrm{n}=43)\end{array}$} & \multicolumn{3}{|c|}{$\begin{array}{c}\text { Difference } \\
\text { between groups }\end{array}$} \\
\hline & $\mathrm{n}$ & Mean $(90 \% \mathrm{Cl})$ & $\mathrm{n}$ & $\operatorname{Mean}(90 \% \mathrm{CI})$ & $(90 \% \mathrm{CI})$ & P value & Effect size \\
\hline \multicolumn{8}{|l|}{ Motor functioning } \\
\hline Motricity Index & 43 & $3.30(0.01,6.59)$ & 42 & $3.74(0.66,6.82)$ & $-0.44(-4.90,4.03)$ & 0.21 & -0.04 \\
\hline Functional Motricity Test & 43 & $0.51(0.04,0.98)$ & 42 & $0.48(0.15,0.80)$ & $0.44(-0.53,0.60)$ & 0.36 & 0.03 \\
\hline \multicolumn{8}{|l|}{ Cognitive functioning } \\
\hline Apraxia & 42 & $6.26(2.76,9.77)$ & 41 & $4.02(0.84,7.21)$ & $2.24(-2.45,6.92)$ & 0.16 & 0.18 \\
\hline \multicolumn{8}{|l|}{ ADL functioning } \\
\hline ADL observations & 39 & $0.21(0.09,0.33)$ & 36 & $0.22(0.10,0.33)$ & $-0.01(-0.17,0.16)$ & 0.37 & -0.02 \\
\hline Barthel ADL Index & 43 & $3.00(1.97,4.03)$ & 40 & $2.83(2.00,3.65)$ & $0.18(-1.14,1.49)$ & 0.11 & 0.05 \\
\hline ADL judgement (OT) & 37 & $1.20(0.79,1.62)$ & 35 & $1.48(1.11,1.85)$ & $-0.27(-0.82,0.28)$ & 0.42 & -0.19 \\
\hline $\mathrm{ADL}$ judgement (Pt) & 37 & $0.72(0.27,1.18)$ & 39 & $0.56(0.28,0.85)$ & $0.16(-0.37,0.68)$ & 0.27 & 0.12 \\
\hline
\end{tabular}

* Analyses are adjusted for age and baseline scores on the specific outcome measures; a one-sided significance test is used. 